\title{
El diagnóstico de situación, una técnica para el análisis de alternativas y la valoración de sus consecuencias
}

\section{Jesús Vilar}

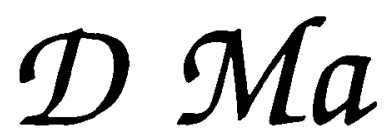

Se consideran las etapas fundamentales para el diagnóstico de situación y los pasos a seguir en su aplicación. Esta técnica que intenta ayudar a los alumnos a pensar, analizar, criticar y juzgar la realidad que les rodea se ejemplifica en actividades dirigidas en especial a los alumnos del segundo ciclo de la Educación Secundaria, ofreciéndose, además, prototipos de actividades para otros tramos educativos.

\section{INTRODUCCION}

El diagnóstico de situación es una técnica de educación moral que tiene por objeto el desarrollo de la capacidad de valorar las diferentes alternativas que se presentan en una situación problemática así como las posibles consecuencias de cada una de ellas, a partir de una situación de diálogo organizado.

En este sentido, el diagnóstico de situación contiene características de tres tipos de procedimientos habitualmente utilizados en educación moral:

- Aquellos que tiene por objeto desarrollar en el sujeto su capacidad de análisis. Conviene distinguir en primer lugar los hechos que se presentan en una situación y las diversas opciones que se barajan, así como los valores que se implican en cada una de ellas.

- Aquellos que tienen por objeto desarrollar el juicio moral. Derivados de la incertidumbre sobre la validez y bondad de la decisión tomada una vez que se analizan las consecuencias de cada decisión y porque el diálogo organizado es una fuente de novedad cuando las opiniones que se presentan son diversas o controvertidas.

- Aquellos que tienen por objeto el desarrollo de la capacidad de llegar a acuerdos mediante el diálogo y la argumentación. El diagnóstico o valoración final requiere que los diferentes miembros del grupo realizen la actividad de 
diagnóstico mediante el uso de la argumentación y la exposición de razones,

las valoren críticamente y lleguen a algún tipo de síntesis.

El punto de partida de esta técnica es, como en los dilemas morales, una situación real o hipotética donde un protagonista ha tomado una decisión sobre una situación problemática de difícil solución, en la que entran en conflicto diversos valores más o menos básicos.

La diferencia fundamental con los dilemas morales es que la técnica de diagnóstico guarda más relación con los contenidos de los valores que se implican en la situación o que se presentan en la materia que se está tratando que con la forma de juicio que se está emitiendo. Es decir, mientras los dilemas se dirigen específicamente a discutir las razones que han movido al protagonista a tomar una decisión determinada, el diagnóstico de situación busca sobre todo que se analice la decisión en sí, que se consideren los valores que se ponen en juego en esa decisión, que se busquen otras alternativas posibles a la decisión que se ha tomado y que se valoren las consecuencias que se derivarían de tomar alguna de ellas.

Por otra parte, esta técnica resulta muy útil para desarrollar la capacidad de análisis de situaciones complejas en las que se deben tener en cuenta un númeo más o menos importante de factores que influyen en esa situación, así como sus posibles consecuencias. Fomenta la habilidad para discernir entre alternativas diversas al considerar las consecuencias que se derivan de cada una de ellas así como los valores que se ponen en juego.-

Esta técnica sigue básicamente tres etapas fundamentales:

- En primer lugar, se presenta una situación conflictiva a los alumnos y se les pide que en grupo o de manera individual busquen criterios para juzgar la situación. En relación con los criterios establecidos, deben pensar otras alternativas posibles que se habrían podido tomar en esa situación.

- En segundo lugar, se valoran las consecuencias de las diferentes alternativas posibles que los alumnos han presentado, en relación con los valores que se ponen en juego en cada una de ellas.

- En tercer lugar, se procede a emitir un juicio para esas situaciones -que será el diagnóstico propiamente dicho,- después de haber discutido colectivamente las repercusiones o consecuencias de la decisión tomada inicialmente o de las decisiones posibles.

Estas tres etapas fundamentales se pueden desglosar en seis pasos:

1. Identificar y clarificar los valores implicados en la situación que se discute. Conviene centrar el problema de manera que queden claros los valores que se ponen en discusión y los argumentos que se aportan.

2. Buscar hechos y datos significativos para el problema que se debate que puedan servir de alternativa a la decisión inicial. Es importante distinguir entre hechos y valores.

3. Comprobar el grado de confianza que nos merecen las posibles alternativas después de considerar los valores que cada una de ellas ponen en juego.

4. Clarificar la relevancia de las alternativas que se han presentado y si son útiles para ayudar a clarificar el problema que se está tratando.

5. Alcanzar una postura consensuada entre todos los miembros del grupo que analizan el tema, a la luz de lo aportado en las etapas anteriores.

6. Evaluar la decisión que se tomó inicialmente viendo si es aceptable en sí misma y en sus consecuencias previsibles. 
En síntesis, podemos decir que esta técnica intenta ayudar a los alumnos a pensar, analizar, criticar y juzgar la realidad que les rodea. También les ayuda a buscar y formar criterios y a emitir juicios estudiando los diferentes niveles en los que se mueve la persona, así como los diversos ángulos de los que se puede enfocar un mismo acontecimiento.

\section{EJEMPLO DE ACTIVIDAD}

La actividad que se pone de ejemplo está seleccionada para personas de 14-16 años aproximadamente y trata el tema de la dificultad que tienen los jóvenes para decidir su futuro cuando se plantean posibilidades muy distantes entre ellas y que afectan la cuestión laboral.

Iremos mostrando como se desarrolla la actividad en relación con los pasos que anteriormente hemos anotado.

\section{Presentación del problema}

Se reparte un texto donde está explicada la situación problemática que se debe diagnosticar. Este texto ha de mostrar claramente cuales son las alternativas que se presentan así como el núcleo del problema. La narración ha de acabar mostrando la decisión que toma el protagonista de la historia que se expone.

También se debe tener preparada una lista de preguntas orientativas para realizar el diagnóstico.

Título de la actividad: «El futuro, el trabajo y el estudio»

«La actividad que os proponemos tiene por objeto que reflexionéis sobre las dudas que se os presentan a la hora de decidir vuestro futuro.» ¿Qué haré? ¿Estudiaré, me dedicaré a trabajar, podré compaginar las dos actividades?»

Para trabajar este tema os proponemos que analicéis esta situación de la que más tarde deberéis hacer un diagnóstico o una valoración. Seguramente, este problema ya se os ha presentado o sabéis de algún amigo o amiga vuestro que se encuentra ante estas dudas.

«Rosa es una chica que estudia $3 .^{\circ}$ de BUP. Nunca ha sido una estudiante excepcional, pero siempre ha trabajado con mucha voluntad y por eso ha obtenido muy buenos resultados en aquellas materias que le gustaban más.

Desde pequeña ha estado muy interesada por el estudio de la naturaleza y hace tiempo que decidió que estudiaría Biológicas en la universidad.

A pesar de su interés por el tema, se ha dado cuenta que cada vez le cuesta más interesarse por el resto de materias de BUP. No se siente motivada y además tiene poco tiempo para estudiar. Esto le está produciendo muchas duchas sobre como plantearse el futuro, sobre todo cuando ve que todavía le queda acabar el curso que está haciendo y todo COU. Además, no toda la biología le interesa por igual y ve que deberán pasar aún algunos años antes de que se pueda dedicar específicamente a aquello que le interesa realmente.

Paralelamente, hace unos meses ha comenzado a trabajar en una oficina durante las tardes. Este trabajo dificulta su concentración y le permite disponer de menos tiempo libre. Además, el otro día le propusieron trabajar todo el día a partir del momento que ella crea conveniente. Es un trabajo monótono que le interesa relativamente, pero es en una empresa sólida y seria, por lo cual puede contar con trabajo fijo y con posibilidades de mejorar su posición laboral. 
Ella es consciente que cada vez está menos interesada por los estudios de bachillerato (aunque le interese la biología) y se está planteando dejar de estudiar para ponerse a trabajar. Esta posibilidad le permitirá tener dinero, tiempo libre y liberarse del nerviosismo y el malestar que le produce la situación en que se encuentra respecto de los estudios. Quizás algún día retomará los estudios. Además, tampoco está claro que en la actualidad una persona pueda trabajar de aquello que ha estudiado.

De todos modos, tiene bastantes dudas. Teme que la seguridad de un trabajo fijo la aleje de lo que realmente le interesa y desea más claramente: estudiar algunos aspectos muy concretos de la naturaleza.

Después de pensarlo mucho, Rosa ha decidido acabar el curso y ponerse a trabajar.»

\section{Como organizarse}

Un aspecto importante es la manera como se organizan los alumnos para garantizar que se realize un trabajo mínimamente correcto y eficaz. No hay que olvidar que la capacidad de trabajo y la motivación del grupo, que es lo que determina en última instancia que se puedan obtener buenos resultados de la actividad, está directamente relacionada con una planificación adecuada de la tarea que se pretende realizar.

En este sentido, conviene tener en cuenta algunos aspectos. En primer lugar la organización del grupo. Conviene hacer grupos pequeños que esten compensados (que todos los miembros del grupo puedan hacer aportaciones y que estas aportaciones generen nuevas dudas entre ellos) y se invita a los alumnos que intenten responder a las preguntas que orientan la realización del diagnóstico.

«Haced grupos de cinco personas. Cada grupo discutirá las preguntas que se anotan a continuación antes de elaborar el diagnóstico definitivo.»

\section{Clasificación de las preguntas orientadoras}

Relacionado con el apartado anterior, otro de los factores importantes a tener en cuenta es que los alumnos no se sientan desconcertados ante las preguntas que deben ir respondiendo y no decrezca el nivel de atención respecto de la tarea.

Así pues, en primer lugar mostraremos algunas preguntas que pueden utilizar la persona que dirige la sesión para orientar la reflexión de los alumnos en el trabajo de diagnóstico. Esas preguntas deben ser consideradas como facilitadoras de la reflexión de los alumnos y serán utilizadas cuando decrezca el nivel de discusión, ya sea porque no se comprende lo que se pide en alguno de los pasos del diagnóstico, ya sea porque hay algún paso que les resulta más difícil de resolver.

Preguntas orientadoras para valorar las alternativas y pensar las consecuencias:

1. ¿Crees que vale la pena estudiar materias que no te interesan durante mucho tiempo, sabiendo que lo que más te interesa tardará mucho en llegar?

2. ¿Crees que vale la pena dedicar demasiados esfuerzos a estudiar si no tienes demasiadas garantías de trabajar en el campo que has estudiado?

3. ¿Crees que los estudios escolares y de BUP. ofrecen lo que los alumnos necesitan?

4. ¿Qué crees que deberían ofrecer los estudios de BUP? 
5. ¿Qué crees que les sobra?

6. ¿Crees que en vuestra edad tenéis el soporte necesario por parte de los adultos con los que tenéis relación? Explica en qué crees que sí y en qué crees que no.

7. ¿Crees que la cuestión económica es decisiva para decidir si continuar estudiando o no? Piensa si no lo es nunca, si depende de las circunstancias o si lo es siempre.

\section{Organización de las preguntas dirigidas a los alumnos}

Cuando se presenta el texto a los alumnos donde se muestra la situación, debemos aportar también el listado de preguntas o actividades que se deben seguir para hacer el diagnóstico. Estas cuestiones deben ir siguiendo, en la medida de lo posible, los pasos que anteriormente anotábamos sobre como realizar el diagnóstico.

Así pues, a continuación mostraremos las preguntas orientadoras que se hacen a los alumnos y las iremos relacionando con los pasos que se deben seguir en la realización del diagnóstico.

Preguntas que se plantean a los alumnos y relación de las mismas con los pasos del diagnóstico:

1. Identificar y clarificar los valores implicados en la situación que se discute.

- Si tu fueras Rosa, ¿qué harías?

- Piensa razones a favor y razones en contra sobre la decisión tomada por Rosa.

- Considerad las consecuencias de esta decisión.

- Pensad qué ha valorado Rosa para tomar esta decisión.

2. Buscar bechos y datos significativos para el problema que se debate que puedan servir de alternativa a la decisión inicial.

- Buscad el núcleo del problema. Realmente, ¿cuál es el problema, cuáles són las variables que lo condicionan y cuáles son los valores que se ponen de manifiesto?

- Una vez habeis encontrado el núcleo del problema y las variables que lo condicionan, pensad alternativas posibles a la decisión que ha tomado Rosa.

- Analizad los valores que se ponen en juego en cada una de las alternativas que habéis pensado, es decir, mostrad qué es lo que se valora con mayor insistencia en cada una de ellas (por ejemplo: paciencia, capacidad de autocontrol y de fijarse objetivos a largo plazo, beneficio económico, estatus social, sinceridad con uno mismo, tranquilidad personal...)

3. Comprobar el grado de confianza que nos merecen las posibles alternativas después de considerar los valores que cada una de ellas ponen en juego.

- Valorad el grado de cada alternativa en función de los valores que se han seguido para proponer las alternativas.

4. Clarificar la relevancia de las alternativas que se han presentado y si son útiles para ayudar a clarificar el problema que se está tratanto.

- En función de las consecuencias que habéis analizado, ordenad las posibles alternativas de la más satisfactoria a la menos satisfactoria. Poned en cada una de ellas lo que se valora con más importancia.

5. Alcanzar una postura consensuada entre todos los miembros del grupo que analizan el tema, a la luz de lo aportado en las etapas anteriores. 


\section{4}

- A partir del trabajo que habeis realizado hasta este momento, haced una propuesta consesuada sobre qué decisión creéis que habría sido más correcta y por qué.

6. Evaluar la decisión que se tomó inicialmente viendo si es aceptable en sí misma $y$ en sus consecuencias previsibles.

- Elaborad un diagnóstico final sobre la decisión que ha tomado Rosa. Justificad el diagnóstico.

\section{Elaboración de una conclusión final}

En el apartado B hemos anotado la necesidad de organizar la clase en pequeños grupos y esta organización la hemos mantenido durante toda la actividad. Para finalizar la actividad, conviene que cada uno de los grupos manifieste las conclusiones a que han llegado y que elaboren conjuntamente, ahora ya todos los alumnos de la clase juntos, una conclusión final donde se muestre el diagnóstico colectivo que se realiza y las razones que lo justifican.

Conviene remarcar que, en general, este tipo de actividades acaban siempre con una conclusión colectiva sobre el trabajo que se ha realizado. El esfuerzo final de llegar a un acuerdo colectivo puede significar que todo el trabajo anterior cobre sentido y sea provechoso. Si queda interrumpido en algún punto, puede ocurrir facilmente que los esfuerzos realizados tanto individualmente como en los pequeños grupos se diluya y se pierda por la razón de no haberse materializado en una conclusión que haga de resumen respecto del tema concreto que se discute como de las habilidades puestas en funcionamiento para la realización del ejercicio.

«Ahora que ya habéis elaborado el diagnóstico, haced una puesta en común de las conclusiones a que cada grupo ha llegado. A continuación haced un mural donde se exponga una conclusión conjunta de toda la clase. Si no hay un acuerdo más o menos amplio, conviene que en el mural se incluyan las opiniones más destacables que hayan aparecido en la discusión final.»

\section{ALGUNOS EJEMPLOS DE DIAGNOSTICO DE SITUACION}

\section{«Juzguemos los casos»}

El Sr. Sumoi es propietario de una empresa immobiliaria. Hace dos años terminó un bloque de 60 viviendas y dos parkings. Los negocios le han ido muy bien pero, esperando que la evolución del mercado de la vivienda fuera todavía positiva, dejó por vender 10 pisos y un parking de 70 plazas. Ahora, al cabo de dos años, los ha puesto a la venta con un incremento de 1,5 millones por cada plaza de parking y tres millones por cada piso.

1. ¿Consideras correcta la actuación de este personaje? ¿Por qué?

2. ¿Qué valores crees que ha considerado para tomar esta decisión?

3. ¿Qué piensas que debería haber hecho? Anota alguna alternativa y los valores que se tienen en cuenta en cada una de ellas.

4. ¿A quién puede repercutir la actuación del Sr. Sumoi? Piensa en las consecuencias de las alternativas que has anotado.

5. ¿Piensas que este personaje ha actuado con profesionalidad? ¿Por qué? 
6. ¿Cuál crees que habría sido la actuación más correcta? ¿Por qué?

7. Haz una valoración final del caso.

\section{«La corrección de exámenes»}

Aurora es profesora de Ciencias Sociales en una escuela. Este fin de semana tiene que corregir todos los exámenes finales de sus alumnos para poder tener entregadas las notas el lunes pero precisamente le han llamado unos amigos, que hace mucho tiempo que no ve, para que vaya con su marido a pasar el fin de semana a la torre que aquellos tienen en Port de la Selva. Ella tiene muchísimas ganas de verlos y de salir, así que le pide a su hija, que está estudiando segundo de historia, que le corrija los exámenes y le ponga las notas.

1. ¿Consideras correcta la actuación de este personaje? ¿Por qué?

2. ¿Qué valores crees que ha considerado a la hora de tomar esta decisión?

3. ¿Qué piensas que debería haber hecho? Apunta algunas alternativas y los valores que se tienen en cuenta en cada una de ellas.

4. ¿A quién puede repercutir la decisión que ha tomado Aurora? ¿En qué sentido? Piensa las consecuencias de las alternativa que has anotado.

5. ¿Piensas que este personaje ha actuado con profesionalidad? ¿Por qué?

6. ¿Cuál crees que habría sido la actuación más correcta? ¿Por qué?

7. Haz una valoración final del caso.

\section{Referencias}

Martinez, M.; Puig, J.M. (1989). Educación y democracia. Laertes. Barcelona.

AA.VV. (1990). Etica i escola. El tractament pedagógic de la diferéncia. Rosa Sensat. Barcelona. 


\section{El diagnóstico de situación, una técnica para}

el análisis de alternativas y la valoración

de sus consecuencias

Jesús Vilar

CL\&E, 1992, 15, pp. 99-105

Resumen: En este artículo se presenta la técnica del diagnóstico de situación, que tiene por objeto el desarrollo de la capacidad de valorar las diferentes alternativas que se presentan en una situación problemática así como las posibles consecuencias de cada una de ellas, a partir de una situación de diálogo organizado.

A continuación se estudian las habilidades que esta técnica puede desarrollar y se localizan las similitudes y diferencias que tiene con los dilemas morales.

Finalmente se muestran las etapas teóricas que se deben seguir para realizar el ejercicio de diagnóstico y se identifican estas etapas en una actividad práctica que sirve de modelo.

Datos sobre el autor: Jesús Vilar es pedagogo y actualmente es becario del Grupo de Investigación en Educación Moral (G.R.E.M.).

Dirección: Departamento de Teoría e Historia de la Educación, Facultad de Pedagogía, Universidad de Barcelona, Baldiri Reixac, s/n 08028 Barcelona. Tel (93) 3333466.

(C) De todos los artículos deberá solicitarse por escrito autorización de CL\&E y de los autores para el uso en forma facsímil, fotocopia o cualquier otro medio de reproducción impresa. CL\&E se reserva el derecho de interponer las acciones legales necesarias en aquellos casos en que se contravenga la ley de derechos de autor.

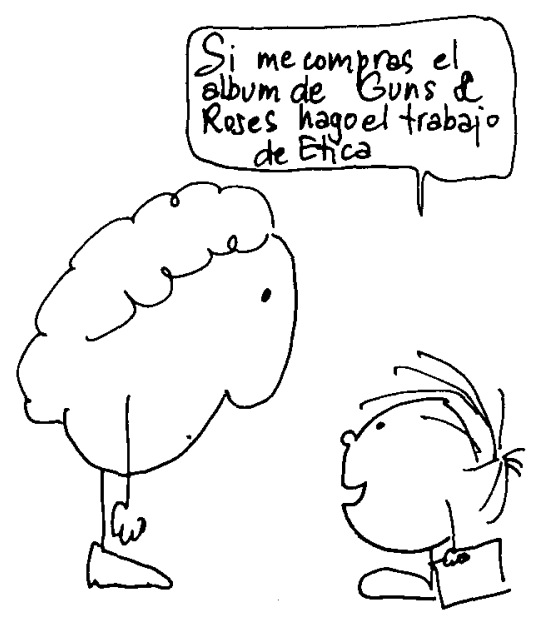

